\title{
ON COMMON FIXED POINTS OF COMPATIBLE MAPPINGS IN METRIC AND BANACH SPACES
}

\author{
S. SESSA \\ Universita di Napoli \\ Facolta di Architettura \\ Istituto Matematico \\ Via Monteoliveto, 3 \\ 80134 Napoli, Italy
}

\section{B.E. RHOADES}

Indiana University

Department of Mathematics

Bloomington, IN 47405

U.S.A.

\section{M.S. KHAN}

King Abdul Aziz University

Faculty of Science

Department of Mathematics

P.O. BOX 9028, Jeddah 21413

Saudi Arabia

(Received on February 16, 1987 and in revised form July 13, 1987)

ABSTRACT. We prove a number of results concerning the existence of common fixed points of a family of maps satisfying certain contractive conditions in metric and Banach spaces. Results dealing with the stucture of the set of common fixed points of such maps are also given. Our work is an improvement upon the previously known results.

KEY WORDS AND PHRASES. As ymptotically regular sequence, Common fixed point, compatible mappings, Strictly convex Banach space.

1980 AMS SUBJECT CLASSIFICATION CODE. 54H25, 47H10

\section{INTRODUCTION}

In [1], we established a number of results concerning common fixed points of three selfmaps on a metric space using sequences which were not necessarily obtained as sequences of iterates of certain maps satisfying Hardy-Rogers [2] type contractive conditions. The maps under consideration also satisfied a condition weaker than commutativity. It is worth noting that, in Jungck [3] and in all generalizations of Jungck's theorem, a family of commuting maps has been considered. More recently, Jungck [4] introduced a further generalization of commutativity. This concept is also more general than that of weak commutativity studied by Sessa [5].

In this paper, we wish to continue our work begun in [1]. Our contractive conditions are motivated by a recent paper of Fisher [6]. We also exploit the idea of compatible pair of maps as used by Jungck [4]. The structure of the set common fixed points in strictly convex Banach spaces is also studied. 
2. PRE:IIIINARIES

Th: s Section contains definitions to be ised in the sequel. Let $A$ and $S$ be two salfinaps of a metric space $(X, d)$. In [1], wa introduced the following definition.

DEFINITION 1. A sequence $\left\{x_{n}\right\}$ in $X$ is sait to be asymptotically A-regular with respect to $S$ if 1 in ${ }_{n} d\left(A x_{n}, S x_{n}\right)=0$.

Further, due to symmetry, we may also siy that $\left\{x_{n}\right\}$ is asymptotically s-regular with respect to A.

La [5], Sessa generalized a result of Das and Vaik [7] using the following definitinn.

DEF[VITION 2. The pair $\{A, S\}$ is sait to be weakly commuting if $d(A S x, S A x)$ $\leqq d(A x, S x)$ for all $x$ in $x$.

A commuting pair is a weakly commuting pair. There are examples in [1] to show that the converse is false.

The following is due to Jungck [4].

DEFIVITION 3. The pair $\{A, S\}$ is said to be compatible if $\lim _{n} \lambda\left(A S x_{n}, S A x_{n}\right)=0$ whenever $\left\{x_{n}\right\}$ is a sequence in $x$ such that $\lim _{n} A\left(x_{n}\right)=\lim { }_{n} S\left(x_{n}\right)=t$ for some point $t$ in $x$.

A we akly commuting pair is a compatible pair. A compatible pair is not necessarily weakly commuting, as is shown in the examples of [4].

\section{COMMON FIXEI) POINTS OF THREE MAPPINGS}

Extending a well known contractive condition of Hardy and Rogers [2], in [1] we considered three selfmaps $A, S$ and $T$ of $(X, d)$ satisfying the following Inequality:

$$
\begin{aligned}
d(A x, A y) & \leq a_{1} \cdot d(S x, A x)+a_{2} \cdot d(T x, A x)+a_{3} \cdot d(S y, A y)+a_{4} \cdot d(T y, A y) \\
& +a_{5} \cdot d(S x, A y)+a_{6} \cdot d(T x, A y)+a_{7} \cdot d(S y, A x) \\
& +a_{8} \cdot d(T y, A x)+a_{9} \cdot d(S x, T y)+a_{10} \cdot d(S y, T x)
\end{aligned}
$$

for all $x, y$ in $x$, where $a_{h}=a_{h}(x, y), h=1, \ldots, 10$, are nonnegative functions of $x^{2}$ into $(0,+\infty)$. Adding to (i) the inequality obtained from (i) by interchanging the roles of $x$ and $y$, we deduce that

$$
\begin{aligned}
d(A x, A y) & <b_{1}(x, y) \cdot d(S x, A x)+b_{1}(y, x) \cdot d(S y, A y)+b_{2}(x, y) \cdot d(T x, A x) \\
& +b_{2}(y, x) \cdot(T y, A y)+b_{3}(x, y) \cdot d(S x, A y)+b_{3}(y, x) \cdot d(S y, A x) \\
& +b_{4}(x, y) \cdot d(T x, A y)+b_{4}(y, x) \cdot d(T y, A x)+b_{5}(x, y) \cdot d(S x, T y) \\
& +b_{5}(y, x) \cdot d(S y, T x)
\end{aligned}
$$

for al1 $x, y$ in $x$, where $b_{j}, j=1, \ldots, 5$, are real nonnegative functions defined by 


$$
\begin{aligned}
& 2 b_{1}(x, y)=a_{1}(x, y)+a_{3}(y, x), 2 b_{2}(x, y)=a_{2}(x, y)+a_{4}(y, x), \\
& 2 b_{3}(x, y)=a_{5}(x, y)+a_{7}(y, x), 2 b_{4}(x, y)=a_{6}(x, y)+a_{8}(y, x), \\
& 2 b_{5}(x, y)=a_{9}(x, y)+a_{10}(y, x)
\end{aligned}
$$

for all $x, y$ in $x$. Seneralizing the well known results of Jungck [3] and Fisher [8], [9], we proved the following result in [1].

THEOREM 1. Let $A, S$ and $T$ be three selfmaps of a complete metric space $(X, d)$ satisfying condition (i) for all $x, y$ in $x$. If

$$
\begin{aligned}
& \max \left\{\sup _{x, y \in X}\left(b_{3}+b_{3}^{\prime}+b_{4}+b_{4}^{\prime}+b_{5}+b_{5}^{\prime}\right),\right. \\
& \sup _{x, y \in X}\left(b_{1}^{\prime}+b_{2}+b_{3}+b_{4}^{\prime}+b_{5}+b_{5}^{\prime}\right), \\
& \left.\sup _{x, y: x}\left(b_{1}^{\prime}+b_{2}^{\prime}+b_{3}+b_{4}\right)\right\}<1,
\end{aligned}
$$

where $b_{1}$ is bounded and $b_{j}^{\prime}(x, y)=b_{j}(y, x)$ ior $j=1, \ldots, 5$ are defined as in (iii), (v) $S$ and $T$ are continuous,

(vi) $\{A, S\}$ and $\{A, T\}$ are weakly commuting pairs,

(vii) there exists an asymptotically A-regular sequence $\left\{x_{n}\right\}$ with respect to both mappings $S$ and $T$, then $A, S$ and $T$ have a unique common fixed point. Further, $A$ is continuous at the fixed point if

$$
\sup _{x, y \in X}\left(b_{1}+b_{2}+b_{3}^{\prime}+b_{4}^{\prime}\right)<1
$$

REMARK 1. In [1], we supposed that $b_{2}$ is also bounded, but this assumption is clearly implied by the condition (iv). Further, it is easily seen that Theorem 1 holds if one assumes the compatibility of the pairs $\{A, S\}$ and $\{A, T\}$ instead of assumption (vi).

By weakening condition (iv), changing ( $v$ ) and (vi), retaining (ii) and (vii), we are able to prove the following result.

THEOREM 2. Let $A, S$ and $T$ be three selfmaps of a complete metric space (X,d) satisfying condition ( $i i)$ for all $x, y$ in $x$, where $b_{j} \geq 0$ and $b_{j}^{\prime}(x, y)=b_{j}(y, x)$ for any $j=1, \ldots, 5$. If

(iv')

$$
\sup _{x, y \in X}\left(b_{3}+b_{3}^{\prime}+b_{4}+b_{4}^{\prime}+b_{5}+b_{5}^{\prime}\right)<1
$$

and $b_{1}, b_{1}^{\prime}, b_{2}, b_{2}^{\prime}$ are bounded,

(v') A is continuous,

(vi') $\{A, S\}$ and $\{A, T\}$ are compatible pairs,

and if ( $v i i$ ) holds, then $A$ has a fixed point.

PROOF. As in [1], we prove that $\left\{A x_{n}\right\}$ is a Cauchy sequence. Indeed, we have on using ( $i i)$,

$$
\begin{gathered}
\left(1-b_{3}-b_{3}^{\prime}-b_{4} b_{4}^{\prime}-b_{5}-b_{5}^{\prime}\right) \cdot d\left(A x_{n}, A x_{m}\right) \leq\left(b_{1}+b_{3}+b_{5}\right) \cdot d\left(S x_{n}, A x_{n}\right) \\
+\left(b_{1}^{\prime}+b_{3}^{\prime}+b_{5}^{\prime}\right) \cdot d\left(S x_{m}, A x_{m}\right)
\end{gathered}
$$




$$
\begin{aligned}
& +\left(b_{2}+b_{4}+b_{5}^{\prime}\right) \cdot J\left(\Gamma x_{n}, A x_{n}\right) \\
& +\left(b_{2}^{\prime}+b_{4}^{\prime}+b_{5}\right) \cdot d\left(\Gamma x_{m}, A x_{m}\right),
\end{aligned}
$$

where $b_{j}=b_{j}\left(x_{n}, x_{m}\right)$ and $b_{j}^{\prime}=b_{j}^{\prime}\left(x_{n}, x_{m}\right)$ for any $j=1, \ldots, 5$. Then we deduce that $i A x_{n}$ ' is a Cauny sequence hy ( $i v^{\prime}$ ) and ( $\left.i i\right)$. Since $X$ is complete,

$\left\{A x_{n}\right\}$ converges to a point $z$. As $d\left(S x_{n}, z\right) \leq d\left(S x_{n}, A x_{n}\right)+d\left(A x_{n}, z\right)$, the sequence $\left\{S x_{n}\right\}$ converges also to $z$. Since $A$ is continuous, the sequences $\left\{A^{2} x_{n}\right\},\left\{A S x_{n}\right\}$ and $\left\{\mathrm{ATx}_{i \mathrm{i}}\right\}$ converge to Az. Using ( $v \mathrm{i}^{\prime}$ ), we obtain

$$
d\left(S A x_{n}, A Z\right) \leq d\left(S A x_{n}, A S x_{n}\right)+d\left(A S x_{n}, A z\right)
$$

which inplies that $\left\{\mathrm{SAx}_{n}\right\}$ converses to Az. Similarly, one proves that $\left\{\right.$ TAx $\left._{n}\right\}$ converges to Az. Further, condition (ii) yields

$$
\begin{aligned}
d\left(A A x_{n}, A x_{n}\right) \leqslant\left(b_{1}+b_{1}^{\prime}+b_{2}+b_{2}^{\prime}\right) \max \left\{d\left(S A x_{n}, A^{2} x_{n}\right), d\left(S x_{n}, A x_{n}\right),\right. \\
\left.d\left(T A x_{n}, A^{2} x_{n}\right), d\left(T x_{n}, A x_{n}\right)\right\} \\
+\left(b_{3}+b_{3}^{\prime}+b_{4}+b_{4}^{\prime}+b_{5}+b_{5}^{\prime}\right) \cdot \max \left\{d\left(S A x_{n}, A x_{n}\right), d\left(S x_{n}, A^{2} x_{n}\right)\right\} \\
\left.d\left(T A x_{n}, A x_{n}\right), d\left(T x_{n}, A^{2} x_{n}\right), d\left(S A x_{n}, T x_{n}\right), d\left(S x_{n}, T A x_{n}\right)\right\}
\end{aligned}
$$

where $b_{j}=b_{j}\left(A x_{7}, x_{n}\right)$ and $b_{j}^{\prime}=b_{j}^{\prime}\left(A x_{n}, x_{n}\right)$ for any $j=1, \ldots, 5$. Then

$$
\begin{aligned}
d(A z, z)= & \text { i imsup }{ }_{n} d\left(A_{n}, A x_{n}\right) \\
< & \limsup _{n}\left(b_{3}+b_{3}^{\prime}+b_{4}+b_{4}^{\prime}+b_{5}+b_{5}^{\prime}\right) \cdot d(A z, z) \\
& \sup \left(b_{3}+b_{3}^{\prime}+b_{4}+b_{4}^{\prime}+b_{5}+b_{5}^{\prime}\right) \cdot d(A z, z), \\
& x, y \in X
\end{aligned}
$$

which means $A z=z$ and thus $z$ is a fixed point of $A$.

REMARK 2. The above theorem assures the existence of a fixed point $z$ of 4 . However, in general, $z$ is not necessarily a fixed point of either $S$ or of $T$ as is shown in the following example.

EXAMPLE 1. Let $X=[0,1]$ with the Euclidean metric d. Define $A, S, T: X+X$ by putting

$$
\begin{aligned}
& A x=x / 16 \text { for all } x \in X, \\
& S x=\left\{\begin{array}{ll}
1 / 16 & \text { if } x=0, \\
x / 16 & \text { if } x \neq 0,
\end{array} \quad T x= \begin{cases}1 / 2 & \text { if } x=0, \\
x / 2 & \text { if } x \neq 0\end{cases} \right.
\end{aligned}
$$


and

$$
\left.d\left(A^{T}\right), T A 0\right)=1 / 2-1 / 32=15 / 32<1 / 2=d(A O, T O) .
$$

Further, $A S x=S A x=x / 256$ and $A T x=T A x=x_{i}^{\prime 32}$ for all $x$ in $X-\{0\}$. Hence $\{A, S\}$ and $\{A, T\}$ are compatible pairs since $A$ weakly conmutes with $S$ and $T$ for all $x$ in $X$. Firthermore,

$$
d(A x, f y)= \begin{cases}0 & \text { if } 0=x=y, \\ \frac{1}{16} y=\frac{1}{8} \cdot \frac{1}{2} y=\frac{1}{3} \cdot d(A x, T y) & \text { if } 0=x<y, \\ \frac{1}{16} x=\frac{1}{8} \cdot \frac{1}{2} x=\frac{1}{8} \cdot d(4 y, T x) & \text { if } 0=y<x, \\ \frac{1}{16}(x-y)=\frac{1}{8} \cdot \frac{1}{2} \cdot(x-y)<\frac{1}{8} \cdot\left(\frac{1}{2} x-\frac{1}{16} y\right)=\frac{1}{8} \cdot d(T x, S y) & \text { if } 0<y<x, \\ \frac{1}{16}(y-x)=\frac{1}{8} \cdot \frac{1}{2}(y-x)<\frac{1}{8}\left(\frac{1}{2} y-\frac{1}{16} x\right)=\frac{1}{8} \cdot d(T y, S x) & \text { if } 0<x<y\end{cases}
$$

Thus condition ( $i i$ ) is satisfied if we assume $b_{1}=b_{2}=b_{3}=0$ and $b_{4}=b_{5}=1 / 8$. Taking a sequence $\left\{x_{n}\right\}, x_{n} \neq 0$ for any positive integer $n$, in $x$ converging to zero, it is easily seen that

$$
\lim m_{n} d\left(A x_{n}, S x_{n}\right)=0 \text { and } \lim m_{n} d\left(A x_{n}, T x_{n}\right)=1 \operatorname{im}_{n} 7 x_{n} / 16=0
$$

Thus all the assumptions of Theorem 2 hold and zero is a fixed point of $A$, but it is not a fixed point of $S$ and of $T$.

The next theorem assures the existence of a fixed point of $S$.

THEOREM 3. Let $A, S$ and $T$ be three selfmaps of a complete metric space (X,d) satisfying condition ( $i i)$ for all $x, y$ in $X$. If conditions (iv') and (vil) hold and $S$ is continuous, then $S$ has a fixed point provided that the pairs $\{A, S\}$ and $\{S, T\}$ are compatible, $b_{j}>0$ and $b_{j}^{\prime}(x, y)=b_{j}(y, x)$ for any $j=1, \ldots, 5$.

PROOF. As in Theorem 2, one shows that the sequences $\left\{A x_{n}\right\},\left\{S x_{n}\right\}$ and $\left\{T_{n}\right\}$ converge to a point $z$. Since $S$ is continuous, the sequences $\left\{S_{A x}\right\},\left\{S^{2} x_{n}\right\}$ and $\left\{S T x_{n}\right\}$ converge to the point $S z$. Using the compatibility of the pairs $\{A, S\}$ and $\{S, T\}$, it is immediately seen that the sequences $\left\{A S x_{n}\right\}$ and $\left\{T S x_{n}\right\}$ converge also to Sz. Now, applying the condition (i $i$ ), we obtain that

$$
\begin{aligned}
d\left(A S x_{n}, A x_{n}\right)< & \left(b_{1}+b_{1}^{\prime}+b_{2}+b_{2}^{\prime}\right) \cdot \max \left\{d\left(S^{2} x_{n}, A S x_{n}\right), d\left(S x_{n}, A x_{n}\right),\right. \\
& \left.d\left(T S x_{n}, A S x_{n}\right), d\left(T x_{n}, A x_{n}\right)\right\} \\
+ & \left(b_{3}+b_{3}^{\prime}+b_{4}+b_{4}^{\prime}+b_{5}+b_{5}^{\prime}\right) \cdot \max \left\{d\left(S^{2} x_{n}, A x_{n}\right), d\left(S x_{n}, A S x_{n}\right),\right. \\
& \left.d\left(T S x_{n}, A x_{n}\right), d\left(T x_{n}, A S x_{n}\right), d\left(S^{2} x_{n}, T x_{n}\right), d\left(S x_{n}, T S x_{n}\right)\right\},
\end{aligned}
$$

where $b_{j}=b_{j}\left(S x_{n}, x_{n}\right)$ and $b_{j}^{\prime}=b_{j}^{\prime}\left(S x_{n}, x_{n}\right)$ for any $j=1, \ldots, 5$. Hence 


$$
\begin{aligned}
1(S z, z)= & 1 \text { imsup }{ }_{n} d\left(\operatorname{ASx}_{n}, S x_{1}\right) \\
\leqslant & 1 \text { imsup }{ }_{n}\left(b_{3}+b_{3}^{\prime}+b_{4}+b_{4}^{\prime}+b_{5}+b_{5}^{\prime}\right) \cdot d(S z, z) \\
\leqslant & \sup \left(b_{3}+b_{3}^{\prime}+b_{4}+b_{4}^{\prime}+b_{5}+b_{5}^{\prime}\right) \cdot d(S z, z)
\end{aligned}
$$

and this gives $S z=z$, i.e. $z$ is a fixed point of $S$.

REMARK 3. A result analogous to Theorem 3 can be obtained using the continitity of $T$ instead of $S$ and the compatibility of the pairs $\{A, T\}$ and $\{S, T\}$.

We now give an example showing that $T$ can have a $f$ ixed point $z$ which is not or fixed point of $A$ and of $S$.

EXAM LF 2. Let $X=[0,1]$ with the Euclidean metric $d$ and $A, S, T: X \rightarrow X$ be defined by $T x=x / 2$ for all $x$ in $X$ and

$$
A x=\left\{\begin{array}{ll}
1 & \text { if } x=0, \\
x / 16 & \text { if } x \neq 0,
\end{array} \quad S x= \begin{cases}1 / 4 & \text { if } x=0 \\
x / 2 & \text { if } x \neq 0\end{cases}\right.
$$

Then we have that

$$
\mathrm{H}(\mathrm{ATO}, \mathrm{TAO})=1-1 / 2=1 / 2<1=\mathrm{d}(\mathrm{AO}, \mathrm{TO}) .
$$

and

$$
\mathrm{d}(\mathrm{STO}, \mathrm{TSO})=1 / 4-1 / 8=1 / 8<1 / 4=\mathrm{d}(\mathrm{SO}, \mathrm{TO}) .
$$

Since $A T x=T A x=x / 32$ and $S T x=T S x=x / 4$ for all $x$ in $X-\{0\}$, we note that $T$ weakly commutes with $A$ and $S$ on the whole space $X$. Hence $\{A, T\}$ and $\{S, T\}$ are compatible pairs and furthermore,

$d(A x, A y)= \begin{cases}0 & \text { if } 0=x=y, \\ 1-\frac{1}{16} y<1=1 \cdot 1=1 \cdot d(A x, T x) & \text { if } 0=x<y, \\ 1-\frac{1}{16} x<1=1 \cdot 1=1 \cdot d(A y, T y) & \text { if } 0=y<x, \\ \frac{1}{16}|x-y|=\frac{1}{8} \cdot \frac{1}{2} \cdot|x-y|=\frac{1}{8} \cdot d(S x, T y) & \text { if } 0 \neq x, 0 \neq y .\end{cases}$

Then condition (ii) holds by assuming $b_{2}=1, b_{5}=1 / 8, b_{1}=b_{3} b_{4}=0$. Taking a sequence $\left\{x_{n}\right\}, x_{n} \neq 0$ for any positive integer $n$, in $x$ converging to zero, we have that

$$
\lim \left(A\left(A x_{n}, S x_{n}\right)=1 i m_{n} d\left(A x_{n}, T x_{n}\right)=1 i m_{n} 7 x_{n} / 16=0\right.
$$

It is clear that $T$ is continuous and $A$ and $S$ are discontinuous at zero, that is a fixed point of $T$, but $A$ and $S$ do not have fixed points.

REMARK 4. If one assumes the additional hypothesis that $S$ (resp. A) is continuous in Theorem 2 (resp. Theorem 3 ), one concludes that $A$ and $S$ have a common fixed point. Indeed, the sequece $\left\{\mathrm{SAx}_{n}\right\}$ (resp. $\left\{\mathrm{ASx}_{n}\right\}$ ) converges to $A z=z$ (resp. $S z=z$ ). 
Since $S\left(\right.$ resp. A) is continuous, the sequence $\left\{\mathrm{SAx}_{n}\right\}$ (resp. $\left\{\mathrm{ASx}_{n}\right\}$ ) converges also to $S z$ (resp. $A z$ ) and then $S z=A z=z$. In general, $z$ is not a $f$ ixed point of $T$, as is shown in the following example.

EXAMPL 3. Let $X=[0,1]$ with the Euclidean metric $d$ and $A, S, T,: \quad X \rightarrow X$ be defined by $A x=x / 16, S x=x / 2$ for all $x$ in $X$ and $T x=1 / 4$ if $x=0, T x=x / 8$ if $x \neq 0$. Since

$$
\mathrm{d}(\mathrm{TAO}, \mathrm{ATO})=1 / 4-1 / 64=15 / 64<1 / 4=\mathrm{d}(\mathrm{AO}, \mathrm{TO})
$$

and $A T x=T A x=x / 128$ for all $x$ in $X-\{0\}$, we $f$ ind that $A$ weakly commutes with $T$ on the whole space $X$, while $A$ commutes with $S$ since $A S x=S A x=x / 32$ for $a l 1 x$ in $X$. Hence condition (vi') holds. Furthermore,

$$
d(A x, A y)= \begin{cases}0 & \text { if } 0=x=y, \\ \frac{1}{16} y=\frac{1}{8} \cdot \frac{1}{2} y=\frac{1}{8} \cdot d(S y, A x) & \text { if } 0=x<y, \\ \frac{1}{16} x=\frac{1}{8} \cdot \frac{1}{2} x=\frac{1}{8} \cdot d(S x, A y) & \text { if } 0=y<x \\ \frac{1}{16}(x-y)=\frac{1}{8} \cdot \frac{1}{2}(x-y)<\frac{1}{8}\left(\frac{1}{2} x-\frac{1}{8} y\right)=\frac{1}{8} d(S x, T y) & \text { if } 0<y<x, \\ \frac{1}{16}(y-x)=\frac{1}{8} \cdot \frac{1}{2}(y-x)<\frac{1}{8}\left(\frac{1}{2} y-\frac{1}{8} x\right)=\frac{1}{8} d(S y, T x) & \text { if } 0<x<y\end{cases}
$$

Then condition ( $i i)$ holds by assuming $b_{3}=b_{5}=1 / 8, b_{1}=b_{2}=b b_{4}=0$. Let $\left\{x_{n}\right\}$ be $a$ sequence in $X$ such that $x_{n} \neq 0$ for any positive integer $n$ and converging to zero. We have that

$$
\lim { }_{n} d\left(A x_{n}, S x_{n}\right)=1 \operatorname{im} 7 x_{n} / 16=0 \text { and } 1 i m_{n} d\left(A x_{n}, T x_{n}\right)=1 \lim _{n} x_{n} / 16=0
$$

Here we point out that $A$ and $S$ are continuous but $T$ is discontinuous at zero. Then all the assumptions of Theorem 2 are satisfied and $A$ and $S$ have zero as common fixed point, but it is not a fixed point of $T$.

Note that

$$
\mathrm{d}(\mathrm{TSO}, \mathrm{STO})=\frac{1}{4}-\frac{1}{8}=\frac{1}{8}<\frac{1}{4}=\mathrm{d}(\mathrm{TO}, \mathrm{SO})
$$

and $T S x=S T x=x / 16$ for all $x$ in $X-\{0\}$. Thus $S$ and $T$ weakly commute and thus all the assumptions of Theorem 3 are also satisfied.

\section{FURTHER EXAMPLES}

of course, if we suppose in Theorem 2 (resp. Theorem 3) additionally the continuity of both mappings $S$ and $T$ (resp. A and $T$ ), we conclude that $z$ is a common fixed point of $A, S, T$ and moreover $z$ is also unique. Indeed, as proved in [1], we have, if $w$ is another common fixed point, using condition (ii),

$$
d(w, z)=d(A w, A z)<\sup _{x, y \in X}\left(b_{3}+b_{3}^{\prime}+b_{4}+b_{4}^{\prime}+b_{5}+b_{5}^{\prime}\right) \cdot d(w, z),
$$


We now give an example of three discontinuous maps which satisfy (ii), but have no fixed points.

EXAMPL 4. Let $X=[0,1]$ with the Euclidean metric $d$ and $A, S, T: X \rightarrow X$ be defined by putting

$$
A x=\left\{\begin{array}{ll}
1 / 8 & \text { if } x=0, \\
x / 8 & \text { if } x \neq 0,
\end{array} \quad \text { if } x=0, \quad \begin{cases}1 & \text { if } x=0 \\
x / 2 & \text { if } x \neq 0,\end{cases}\right.
$$

Now,

$$
\begin{aligned}
& d(\text { ASO }, \text { SAO })=\frac{1}{8}-\frac{1}{16}=\frac{1}{16}<\frac{7}{8}=1-\frac{1}{8}=d(S O, A O), \\
& d(\text { TSO, STO })=\frac{1}{2}-\frac{1}{4}=\frac{1}{4}<\frac{1}{2}=1-\frac{1}{2}=d(S O, \text { TO }),
\end{aligned}
$$

$\mathrm{ATO}=\mathrm{TAO}=1 / 16$ and $\mathrm{ATx}=\mathrm{TAx}=\mathrm{ASx}=\mathrm{SAx}=\mathrm{x} / 16, \mathrm{STx}=\mathrm{TSx}=\mathrm{TSx}=\mathrm{x} / 4$ for all $\mathrm{x}$ in $\mathrm{x}-\{0\}$. Thus each of the pairs $\{A, S\},\{S, T\}$ and $\{A, T\}$ is compatible. Further, we have that

$d(A x, A y)= \begin{cases}0 & \text { if } 0=x=y, \\ \frac{1}{8} \cdot(1-y)<\frac{1}{4} \cdot\left(1-\frac{1}{2} y\right)=\frac{1}{4} \cdot d(S x, T y) & \text { if } 0=x<y, \\ \frac{1}{8}(1-x)<\frac{1}{4} \cdot\left(1-\frac{1}{2} x\right)=\frac{1}{4} \cdot d(S y, T x) & \text { if } 0=y<x, \\ \frac{1}{8}|x-y|=\frac{1}{4} \cdot \frac{1}{2} \cdot|x-y|=\frac{1}{4} \cdot d(S x, T y) & \text { if } 0 \neq x, 0 \neq y .\end{cases}$

By choosing a sequence $\left\{x_{n}\right\}, x_{n} \neq 0$ for any positive integer $n$, in $x$ converging to zero, we deduce that

$$
11 m_{n} d\left(A x_{n}, S x_{n}\right)=1 i m_{n} d\left(a x_{n}, T x_{n}\right)=11 m_{n} 3 x_{n} / 8=0
$$

By assuming $b_{1}=b_{2}=b_{3}=b_{4}=0, b_{5}=1 / 4$ we find that all the assumptions of Theorems $1,2,3$ hold except the continuity of $A, S$ and $T$. Here none of the mappings has a fixed point.

The condition of compatibility is also necessary in Theorems 1,2 and 3 , as is shown in the following example:

EXAMPLE 5. Let $X=[0,+\infty)$ with the Euclidean metric $d$ and define $A, S=T$ : $X \rightarrow X$ by setting

$$
A x=\frac{1}{8} x+1 \quad \text { and } \quad S x=\frac{1}{2} x+1
$$

for all $x$ in $X$. Obviously the sequences $\left\{A x_{n}\right\}$ and $\left\{S x_{n}\right\}$ converge to one iff $\left\{x_{n}\right\}$ converges to zero, but

$$
1 \operatorname{im}_{n} d\left(\operatorname{ASx}_{n}, S A x_{n}\right)=\frac{1}{2}-\frac{1}{8}=\frac{3}{8}
$$

So $\{A, S\}$ is not a compatible pair and further, we have that 


$$
d(A x, A y)=\frac{1}{8} \cdot|x-y|=\frac{1}{4} \cdot \frac{1}{2} \cdot|x-y|=\frac{1}{4} \cdot d(S x, S y)
$$

for all $x, y$ in $X$ and

$$
\lim { }_{n} d\left(A x_{n}, S x_{n}\right)=1 \operatorname{im}_{n} \frac{3}{8} x_{n}=0 \text { iff } 1 \text { im } x_{n}=0
$$

Now, by choosing a sequence $\left\{x_{n}\right\}$ in $x$ converging to zero and by taking $b_{1}=b_{2}=b_{3}=b_{4}=0, b_{5}=1 / 4$, we see that all the assumptions of Theorems 1,2 and 3 hold except the compatibility of the pair $\{A, S\}$. Clearly, A and $S$ do not have common fixed points.

The condition (vii) is also necessary in Theorems 1,2,3. We show this in the next example.

EXAMPLE 6. Let $X=[1,+\infty)$ with the Euclidean metric $d$ and consider $A$ and $S=T$ as in Example 5. Then

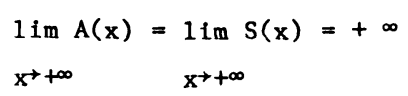

and this means that $A$ and $S$ do not converge to an element $t$ of $X$. Then the condition of compatibility is satisfied vacuosly, but condition ( $v i i$ ) does not hold because the point zero does not belong to $X$. Thus all the assumptions of Theorems 1,2,3 hold except condition (vii) but $A$ and $S$ do not have common fixed points.

\section{FIXED POINTS OF FOUR MAPPINGS}

Recently, Fisher [6] partially extended the result of [7] to obtain the following theorem:

THEOREM 4. Let $A, B, S$ and $T$ be four selfmaps of a complete metric space (X,d) satisfying the inequality

$$
d(A x, B y)<c \cdot \max \{d(A x, S x), d(B y, T y), d(S x, T y)\}
$$

for all $x, y$ in $x$, where $0 \leq c<1$. If

(ix) one of $A, B, S$ and $T$ is continuous,

$(x)\{A, S\}$ and $\{B, T\}$ are commuting pairs,

( $x i) B(X) \subseteq S(X)$ and $A(X) \subseteq T(X)$,

then $A, B, S$ and $T$ have a unique common fixed point $z$. Further, $z$ is the common unique fixed point of $A$ and $S$ and of $B$ and $T$.

REMARK 5. As pointed out by Massa [10], condition (viii) is equivalent to the following

$$
d(A x, B y)<a_{1} \cdot d(A x, S x)+a_{2} \cdot d(B y, T y)+a_{3} \cdot d(S x, T y)
$$

for all $x, y$ in $x$, where $a_{h}=a_{h}(x, y), h=1,2,3$ are nonnegative functions from $x^{2}$ into $[0,+\infty)$ such that

$$
\sup _{x, y \in X}\left(a_{1}+a_{2}+a_{3}\right)<1
$$

The above remark and some recent contractive conditions involving four mappings 
studied by several authors, e.g. Chang [11], Fisher and Sessa [12], Kasahara and Singh [13] lead to the following extension of (vi1i):

$$
\begin{gathered}
d(A x, B y)<a_{1} \cdot d(A x, S x)+a_{2} \cdot d(B y, T y)+a_{3} \cdot d(S x, B y) \\
+a_{4} \cdot d(T y, A x)+a_{5} \cdot d(S x, T y)
\end{gathered}
$$

for all $x, y$ in $x$, where $a_{h}=a_{h}(x, y)$ for any $h=1, \ldots 5$. It is not hard to prove the following generalization of Theorem 4 using ideas from [12].

THEOREM 5. Let $A, B, S$ and $T$ be four selfmaps of a complete metric space $(X, d)$ satisfying condition $(x i i)$ for all $x, y$ in $x$. If $a_{h}>0$ for any $h=1, \ldots, 5$ and

$$
\sup _{x, y \in X}\left(a_{1}+a_{2}+2 a_{3}+2 a_{4}+a_{5}\right)<1,
$$

$\left(\mathrm{X}^{\prime}\right)\{\mathrm{A}, \mathrm{S}\}$ and $\{\mathrm{B}, \mathrm{T}\}$ are compatible pairs

and $(i x),(x i)$ hold, then $A, B, S$ and $T$ have a unique common fixed point, which is unique common fixed point of $A$ and $S$ and of $B$ and $T$.

REMARK 6. Chang [11], studying an analogous contractive condition, assumes the continuity of both mappings $S$ and $T$, the commutativity of the pairs $\{A, S\},\{A, T\}$, $\{B, S\},\{B, T\}$ and

$\left(x 1^{\prime}\right)$

$$
A(X) \cup B(X) \subseteq S(X) \cap T(X)
$$

It is immediately seen that Chang's result holds under the weaker assumptions (ix), ( $\left.x^{\prime}\right)$ and ( $\left.x i\right)$. Clearly ( $\left.x i\right)$ is more general than ( $\left.x i^{\prime}\right)$ since

$$
B(X) \subseteq A(X) \cup B(X) \subseteq S(X) \cap T(X) \subseteq S(X)
$$

and

$$
A(X) \subseteq A(X) \cup B(X) \subseteq S(X) \cap T(X) \subseteq T(X)
$$

REMARK 7. Theorem 5 can also be seen as a special case of Theorem 1 of [14]. Using the mainideas of the present work and those contained in a recent paper due to Rhoades and Sessa [15], we establish another result replacing the condition ( $x i$ ) by the existence of two asymptotically regular sequences, by weakening condition ( $x$ ) and imposing an additional condition.

THEOREM 6. Let $A, B, S$ and $T$ be four selfmaps of a complete metric space $(X, d)$ satisfying condition ( $x i 1$ ) for all $x, y$ in $x$. Further, suppose that $a_{h}>0$ for any $h=1, \ldots .5$ and

$$
\max \left\{\sup _{x, y \in X}\left(a_{3}+a_{4}+a_{5}\right), \sup _{x, y \in X}\left(a_{1}+a_{4}\right), \sup _{x, y \in X}\left(a_{2}+a_{3}\right)\right\}<1,
$$

(xiv) S is continuous,

(xv) $d(x, T x) \leq d(x, S x)$ for a11 $x$ in $x$,

(xvi) $\{A, S\}$ is a compatible pair, 
(xvii) there exist an asymptotically A-regular sequence $\left\{x_{n}\right\}$ with respect to $S$ and an as ymptotically $B$-regular sequence $\left\{y_{n}\right\}$ with respect to $T$.

Then $A, B, S$ and $T$ have a unique common fixed point, which is also a unique common fixed point of $A$ and $S$ and of $B$ and $T$.

PROOF. From (xii), we have for any positive integers $m, n$ :

$$
\begin{aligned}
d\left(A x_{m}, B y_{n}\right) & <a_{1} \cdot d\left(A x_{m}, S x_{m}\right)+a_{2} \cdot d\left(B y_{n}, T y_{n}\right) \\
& +a_{3} \cdot\left[d\left(A x_{m}, S x_{m}\right)+d\left(A x_{m}, B y_{n}\right)\right] \\
& +a_{4} \cdot\left[d\left(B y_{n}, T y_{n}\right)+d\left(A x_{m}, B y_{n}\right)\right] \\
& +a_{5} \cdot\left[d\left(A x_{m}, S x_{m}\right)+d\left(A x_{m}, B y_{n}\right)+d\left(B y_{n}, T y_{n}\right)\right],
\end{aligned}
$$

where $a_{j}=a_{j}\left(x_{m}, y_{n}\right)$ for any $j=1, \ldots, 5$. Then

$$
\begin{aligned}
\left(1-a_{3}-a_{4}-a_{5}\right) \cdot d\left(A x_{m}, B y_{n}\right) & <\left(a_{1}+a_{3}+a_{5}\right) \cdot d\left(A x_{m} \cdot S x_{m}\right) \\
& +\left(a_{2}+a_{4}+a_{5}\right) d\left(B y_{n}, T y_{n}\right),
\end{aligned}
$$

Since

$$
d\left(A x_{m}, A x_{n}\right)<d\left(A x_{m}, B y_{n}\right)+d\left(A x_{n}, B y_{n}\right)
$$

we obtain the inequality

$$
\begin{aligned}
& d\left(A x_{m}, A x_{n}\right)<\frac{\left(a_{1}+a_{3}+a_{5}\right) \cdot d\left(A x_{m}, S x_{m}\right)}{1-\sup _{x, y \varepsilon X}\left(a_{3}+a_{4}+a_{5}\right)} \\
& +\frac{\left(a_{1}^{\prime}+a_{3}^{\prime}+a_{5}^{\prime}\right) \cdot d\left(A x_{n}, S x_{n}\right)}{1-\sup _{x, y \in X}\left(a_{3}^{\prime}+a_{4}^{\prime}+a_{5}^{\prime}\right)} \\
& +\left[\frac{a_{2}+a_{4}+a_{5}}{1-\sup _{x, y \in X}\left(a_{3}+a_{4}+a_{5}\right)}+\frac{a_{2}^{\prime}+a_{4}^{\prime}+a_{5}^{\prime}}{1-\sup _{x, y \varepsilon X}\left(a_{3}^{\prime}+a_{4}^{\prime}+a_{5}^{\prime}\right)}\right] d\left(B y_{n}, T y_{n}\right),
\end{aligned}
$$

where $a_{j}=a_{j}\left(x_{m}, y_{n}\right)$ and $a_{j}^{\prime}=a_{j}\left(x_{n}, y_{n}\right)$ for any $j=1, \ldots, 5$. Now by (xvi $\left.i\right)$, we deduce that $\left\{A x_{n}\right\}$ is a Cauchy sequence, which has a limit z (say). As

$$
d\left(S x_{n}, z\right)<d\left(A x_{n}, S x_{n}\right)+d\left(A x_{n}, z\right)
$$

we see that the sequence $\left\{S x_{n}\right\}$ also converges to $z$. Similarly, it can be proved that the sequences $\left\{B y_{n}\right\}$ and $\left\{T_{n}\right\}$ also converge to $z$. By (xiv), both the sequences $\left\{s^{2} x_{n}\right\}$ and $\left\{S A x_{n}\right\}$ converge to $S z$. By condition (xvi), the sequence $\left\{A S x_{n}\right\}$ also converges to $\mathrm{Sz}$. 
Again by ( $x i i)$, we have

$$
\begin{aligned}
& d\left(\operatorname{ASx}_{n}, B y_{n}\right)<\left(a_{1}+a_{2}\right) \cdot \max \left\{d\left(A S x_{n}, s^{2} x_{n}\right), d\left(B y_{n}, T y_{n}\right)\right\} \\
&+\left(a_{3}+a_{4}+a_{5}\right) \cdot \max \left\{d\left(S^{2} x_{n}, B y_{n}\right), d\left(A S x_{n}, T y_{n}\right),\right. \\
&\left.d\left(S^{2} x_{n}, T y_{n}\right)\right\},
\end{aligned}
$$

where $a_{j}=a_{j}\left(S x_{n}, y_{n}\right)$ for any $j=1, \ldots, S$. Hence

$$
\begin{aligned}
d(S z, z) & =1 \text { imsup } \\
& d\left(A S x_{n}, B y_{n}\right) \\
& <\limsup _{n}\left(a_{3}+a_{4}+a_{5}\right) \cdot d(S z, z) \\
& <\sup _{x, y \in X}\left(a_{3}+a_{4}+a_{5}\right) \cdot d(S z, z),
\end{aligned}
$$

whence $S z=z$ by (xiii).

Due to $(x i 1)$, we obtain

$$
\begin{aligned}
d\left(A z, B y_{n}\right)< & \left(a_{1}+a_{4}\right) \cdot \max \left\{d(A z, S z), d\left(T y_{n}, A z\right)\right\} \\
& +\left(a_{2}+a_{3}+a_{5}\right) \cdot \max \left\{d\left(B y_{n}, T y_{n}\right), d\left(S z, B y_{n}\right), d\left(S z, T y_{n}\right)\right\},
\end{aligned}
$$

where $a_{j}=a_{j}\left(z, y_{n}\right)$ for any $j=1, \ldots, 5$. Then

$$
\begin{aligned}
d(A z, z)=1 \operatorname{lisup}_{n} d\left(A z, B y_{n}\right) & \leqslant \limsup \left(a_{1}+a_{4}\right) \cdot d(A z, z) \\
& \leqq \sup _{x, y \in X}\left(a_{1}+a_{4}\right) \cdot d(A z, z),
\end{aligned}
$$

which implies $A z=z$ by (xili). From (xv), we deduce that

$$
d(z, T z)<d(z, S z)=0,
$$

i.e. Tz=z. Further, ( $x i 1)$ implies that

$$
\begin{aligned}
d(z, B z)=d(A z, B z) & <a_{1} \cdot d(A z, S z)+a_{2} \cdot d(B z, T z) \\
& +a_{3} \cdot d(S z, B z)+a_{4} \cdot d(T z, A z)+a_{5} \cdot d(S z, T z) \\
& <\sup _{x, y \in X}\left(a_{2}+a_{3}\right) \cdot d(z, B z),
\end{aligned}
$$

where $a_{j}=a_{j}(z, z)$ for any $j=1, \ldots, 5$. By ( $\left.x i 1 i\right)$, we have that $B z=z, 1 . e$. $z$ is a fixed point of $A, B, S$ and $T$. Let $w$ be another common fixed point of $A$ and $S$. From (xii), we get 


$$
\begin{aligned}
d(w, z)=d(A w, B z)< & a_{1} \cdot d(A w, S w)+a_{2} \cdot d(B z, T z) \\
+ & a_{3} \cdot d(S w, B z)+a_{4} \cdot d(\Gamma z, A w)+a_{5} \cdot d(S w, T z) \\
& <\sup _{x, y \in X}\left(a_{3}+a_{4}+a_{5}\right) \cdot d(z, w),
\end{aligned}
$$

where $a_{j}=a_{j}(w, z)$ for any $j=1, \ldots, 5$. So $z=w$ by (xiii) and similarly, one would prove that $z$ is the unique common fixed point of $B$ and $T$. This completes proof.

\section{SOME REMARKS ON THEOREM 6 .}

REMARK 8. A result analogous to Theorem 6 can be formulated supposing $T$ continuous, $" d(x, S x)<d(x, T x)$ for all $x$ in $X "$ and requiring the pair $\{B, T\}$ to be compatible.

REMARK 9. The sum $\left(a_{1}+a_{2}+a_{3}+a_{4}+a_{5}\right)$ may exceed 1 , in contrast to Hardy and Rogers [2] where $\left(a_{1}+a_{2}+a_{3}+a_{4}+a_{5}\right)<1$.

REMARK 10. Note that if one does not assume the condition "d(x,Tx)< d(x,Sx) for all $x$ in $X^{\prime \prime}$ in Theorem 6, then this theorem need no longer be true. Examining the proof of Theorem 6, one concludes only that $S$ and $A$ have a common fixed point $z$, but in general, $z$ need not be a fixed point of either $A$ or of $T$. This is seen in the following example borrowed from Fisher [6].

EXAMPLE 7. Let $X=[0,1]$ with the Euclidean metric $d$ and define $A x=0, S x=x$ for a $11 \times$ in $X$ and

$$
B x=\left\{\begin{array}{ll}
1 / 4 & \text { if } x=0, \\
x / 4 & \text { if } x \neq 0,
\end{array} \quad T x= \begin{cases}1 & \text { if } x=0 \\
x & \text { if } x \neq 0\end{cases}\right.
$$

Note that A commutes with $S$ and $S$ is continuous, whereas $d(0, T 0)=1>0=d(0, S 0)$. Thus condition ( $x v$ ) is not satisfied at the point zero. Further, we have for all $x$ in $\mathrm{X}$,

$$
d(A x, B y)= \begin{cases}\frac{1}{4}=\frac{1}{3} \cdot \frac{3}{4}=\frac{1}{3} \quad\left(1-\frac{1}{4}\right)=\frac{1}{3} \cdot d(B O, T O) & \text { if } y=0, \\ \frac{1}{4} y=\frac{1}{3} \cdot \frac{3}{4} y=\frac{1}{3} \cdot\left(y-\frac{1}{4} y\right)=\frac{1}{3} \cdot d(B y, T y) & \text { if } y \neq 0 .\end{cases}
$$

For any sequence $\left\{x_{n}\right\}$ converging to zero, we have that

$$
\lim _{n} d\left(A x_{n}, S x_{n}\right)=11 m_{n} x_{n}=0
$$

and, by choosing a sequence $\left\{y_{n}\right\}, y_{n} \neq 0$ for any positive integer $n$, we get

$$
\lim { }_{n} d\left(B y_{n}, T y_{n}\right)=1 i m_{n} \frac{3}{4} y_{n}=0
$$

Then all the assumptions of Theorem 6 are satisfled with $a_{1}=a_{3}=a_{4}=a_{5}=0$ and $a_{2}=1 / 3$, except condition $(x v)$, and zero is the common fixed point of $A$ and $S$ but zero is not a fixed point of $B$ and of $T$. 
REMARK 11. If, in Theorem 6, one also assumes the continuity of $T$, the compatibility of the pair $\{B, T\}$ and hypothesis (xv) is dropped, then the conclusion of Theorem 6 still holds. Note that this remark does not apply to Example 7 , where, even if $B$ commutes with $T, T$ is discontinuous at zero.

Assuning the continuity of $A$, the following result holds.

THEOREM 7. Let $A, B, S$ and $T$ be four selfmaps of a complete metric space satisfying condition ( $x i 1$ ) for $a l 1 x, y$ in $x$. If $a_{h} \geq 0$ for any $h=1, \ldots, 5$ and

(xviii)

$$
\sup _{x, y \in X}\left(a_{3}+a_{4}+a_{5}\right)<1
$$

if $a_{1}, a_{2}$ are bounded and if (xvi), (xvii) hold, then $A$ has a fixed point provided that $A$ is continuous.

PROOF. As in the proof of Theorem 6, one proves that the sequences $\left\{A x_{n}\right\},\left\{S x_{n}\right\},\left\{B y_{n}\right\},\left\{T y_{n}\right\}$ converge to a point $z$. Since $A$ is continuous, the sequences $\left\{A^{2} x_{n}\right\}$ and $\left\{A S x_{n}\right\}$ converge to $A z$. Using ( $\left.x v i\right)$, it is seen that the sequence $\left\{\mathrm{SAx}_{\mathrm{n}}\right\}$ converges to $\mathrm{Az}$. Then,

$$
\begin{aligned}
d\left(\operatorname{AAx}_{n}, B y_{n}\right) & <\left(a_{1}+a_{2}\right) \cdot \max \left\{d\left(A^{2} x_{n}, S A x_{n}\right), d\left(B y_{n}, T y_{n}\right)\right\} \\
& +\left(a_{3}+a_{4}+a_{5}\right) \cdot \max \left\{d\left(S A x_{n}, B y_{n}\right), d\left(T y_{n}, A^{2} x_{n}\right), d\left(S A x_{n}, T y_{n}\right)\right\},
\end{aligned}
$$

where $a_{j}=a_{j}\left(A x_{n}, y_{n}\right)$ for any $j=1, \ldots, 5$. Then

$$
\begin{aligned}
d(A z, z)=1 \operatorname{lisup}_{n} d\left(A^{2} x_{n}, B y_{n}\right) & <\operatorname{limsup_{n}}\left(a_{3}+a_{4}+a_{5}\right) \cdot d(A z, z) \\
& <\sup _{x, y \in X}\left(a_{3}+a_{4}+a_{5}\right) \cdot d(A z, z),
\end{aligned}
$$

giving thereby $A z=z$.

REMARK 12. Theorem 7 assures the existence of a fixed point $z$ of $A$, but in general $z$ is not a fixed point of $B, S$ and $T$ as is shown in the following example.

EXAMPLE 8. Let $x=[0,1]$ with the Euclidean metric $d$ and define $A x=x / 8$ for all $x$ In $X$. Define $B, S, T: X+X$ as

$$
B X=\left\{\begin{array}{ll}
1 / 2 & \text { if } x=0, \\
x / 4 & \text { if } x \neq 0,
\end{array} \quad S x=\left\{\begin{array}{ll}
1 & \text { if } x=0, \\
x / 2 & \text { if } x \neq 0,
\end{array} \quad \text { Tx }= \begin{cases}1 & \text { if } x=0, \\
x & \text { if } x \neq 0\end{cases}\right.\right.
$$

Since

$$
\mathrm{d}(\mathrm{ASO}, \mathrm{SAO})=1-\frac{1}{8}=\frac{7}{8}<1=\mathrm{d}(\mathrm{AO}, \mathrm{SO})
$$

and $S A x=A S x=x / 16$ for $a 11 x$ in $X-\{0\}, A$ is weakly commuting with $S$ on the whole space $X$ and hence condition ( $x v i$ ) holds. Note that $A$ is continuous and condition (xvi $i$ ) is satisfied by choosing sequences $\left\{x_{n}\right\}$ and $\left\{y_{n}\right\}, x_{n} \neq 0$ and $y_{n} \neq 0$ for any positive integer $n$, both converging to zero. Indeed, we have,

$$
\lim m_{n} d\left(A x_{n}, S x_{n}\right)=11 m_{n} 3 x_{n} / 8=0,11 m_{n} d\left(B y_{n}, T y_{n}\right)=11 m_{n} 3 y_{n} / 4=0
$$


Now we verify condition (xii). We have

$$
d(A x, B y)= \begin{cases}\frac{1}{2}=1 \cdot \frac{1}{2}=1 \cdot d(B y, T y) & \text { if } x=y=0, \\ \frac{1}{2}-\frac{1}{8} x=\frac{1}{2}\left(1-\frac{1}{4} x\right)<\frac{1}{2}\left(1-\frac{1}{8} x\right)=\frac{1}{2} \cdot d(T y, A x) & \text { if } x \neq 0, y=0, \\ \frac{1}{4} y<\frac{1}{2} y=\frac{1}{2} \cdot d(T y, A x) & \text { if } x=0, y \neq 0, \\ \left|\frac{x}{8}-\frac{y}{4}\right|=\frac{1}{4} \cdot\left|\frac{x}{2}-y\right|=\frac{1}{4} \cdot d(S x, T y) & \text { if } x \neq 0, y \neq 0 .\end{cases}
$$

Then (xvili) holds by assuming $a_{1}=a_{3}=0, a_{2}=1, a_{4}=1 / 2, a_{5}=1 / 4$. Thus all the conditions of Theorem 7 hold and zero is a fixed point of $A$ but it is not a fixed point of $B, S$ and $T$.

6. RESULTS IN BANACH SPACES.

We first prove the following Lemma.

LEMMA. Let $(X, d)$ be a complete metric space and $K$ be a closed subset of $X$. Let $A, B, S$ and $T$ be four selfmaps of $K$ satisfying condition ( $x i 1$ ) for all $x, y$ in $K$. If $a_{h}>0$ for any $h=1, \ldots, 5$,

$\left(x 111^{\prime}\right)$

$$
\max \left\{\sup _{x, y \in X}\left(a_{1}+a_{4}\right), \sup _{x, y \in X}\left(a_{2}+a_{3}\right)\right\}<1
$$

$a_{5}$ is bounded, ( $\left.x i v\right)$ and (xv) hold, then the set $F$ of the common fixed points of $A, B, S$ and $T$ is closed.

PROOF. Let $\left\{x_{n}\right\}$ be a Cauchy sequence in $F$ with limft $x$ in $K$.

Then, from ( $x i 1)$

$$
\begin{aligned}
d(x, A x,) & \leqslant d\left(x_{n}, x\right)+d\left(A x, B x_{n}\right) \\
& <d\left(x_{n}, x\right)+a_{1} \cdot d(A x, S x)+a_{2} \cdot d\left(B x_{n}, T x_{n}\right) \\
& +a_{3} \cdot d\left(S x, B x_{n}\right)+a_{4} \cdot d\left(A x, T x_{n}\right)+a_{5} \cdot d\left(S x, T x_{n}\right) \\
& <\left(1+a_{4}\right) \cdot d\left(x_{n}, x\right)+a_{1} \cdot\left[d(x, A x)+d\left(x_{,} x_{n}\right)+\left(x_{n}, S x\right)\right] \\
& +a_{4} \cdot d(x, A x)+\left(a_{3}+a_{5}\right) \cdot d\left(x_{n}, S x\right),
\end{aligned}
$$

where $a_{j}=a_{j}\left(x, x_{n}\right)$ for any $j=1, \ldots, 5$. So

$$
\left(1-a_{1}-a_{4}\right) \cdot d(x, A x)<\left(1+a_{1}+a_{4}\right) \cdot d\left(x_{n}, x\right)+\left(a_{1}+a_{3}+a_{5}\right) \cdot d\left(x_{n}, S x\right) \cdot
$$

Since

$$
d\left(x_{n}, S x\right)=d\left(S x_{n}, S x\right),
$$




$$
d(x, S x)<d\left(x, x_{n}\right)+d\left(S x_{n}, S x\right)
$$

we have $x=S x$. From ( $x v), x=T x$. Using ( $x i 1$ ) again, we deduce that

$$
\begin{aligned}
d(x, B x)=d(A x, B x) & \leqslant a_{1} \cdot d(A x, S x)+a_{2} \cdot d(B x, T x) \\
& +a_{3} \cdot d(S x, B x)+a_{4} \cdot d(T x, A x)+a_{5} \cdot d(S x, T x) \\
& =\left(a_{2}+a_{3}\right) \cdot d(x, B x),
\end{aligned}
$$

where $a_{j}=a_{j}(x, x)$ for any $j=1, \ldots, 5$. By $\left(x 111^{\prime}\right), x=B x$ and hence $F$ is closed.

THEOREM 8. Let $X$ be a strictly convex Banach space, $K$ be a closed convex subset of $X, A, B, S, T$ be four selfmaps of $K$ satisfying condition ( $x i 1$ ) for all $x, y$ in $K$. If

(x1i1")

$$
\sup _{x, y \in X}\left(a_{1}+a_{3}+a_{4}+a_{5}\right)<1
$$

( $\left.x i i i^{\prime}\right),(x i v)$ and ( $x v$ ) hold, then the set $F$ of the common fixed points of $A, B, S$ and $T$ is closed and convex provided that $S$ is linear and $a_{h}>0$ for any $h=1, \ldots, 5$.

PROOF. Since (xili") implies that $a_{5}$ is bounded, $F$ is closed by the Lemma. Let $x_{1}, x_{2}$ be points of $F$ and $x=\left(x_{1}+x_{2}\right) / 2$. Since $K$ is convex, $x$ belongs to $K$. Since $S$ is linear, we have

$$
S x=\frac{S x_{1}+S x_{2}}{2}=\frac{x_{1}+x_{2}}{2}=x,
$$

i.e. $x$ is a fixed point of $S$. Without loss of generality, we may assume that

$$
\left\|x_{2}-A x\right\| \leq\left\|x_{1}-A x\right\|
$$

Then

$$
\|S x-A x\|=\|x-A x\|<\frac{1}{2} \cdot\left[\left\|x_{1}-A x\right\|+\left\|x_{2}-A x\right\|\right]<\left\|x_{1}-A x\right\| \text {. }
$$

Using ( $x 11)$, we get

$$
\begin{aligned}
& \left\|x_{1}-A x\right\|=\left\|A x-B x_{1}\right\|<a_{1} \cdot\|A x-S x\|+a_{2} \cdot\left\|B x_{1}-T x_{1}\right\| \\
& +a_{3} \cdot\left\|S x-B x_{1}\right\|+a_{4} \cdot\left\|T x_{1}-A x\right\|+a_{5} \cdot\left\|S x-T x_{1}\right\| \\
& <\left(a_{1}+a_{4}\right) \cdot\left\|x_{1}-A x\right\|+\left(a_{3}+a_{5}\right) \cdot\left\|x-x_{1}\right\|
\end{aligned}
$$

where $a_{j}=a_{j}\left(x, x_{1}\right)$ for any $j=1, \ldots, 5$. This implies that

$$
\left(1-a_{1}-a_{4}\right) \cdot\left\|x_{1}-A x\right\|<\left(a_{3}+a_{5}\right) \cdot\left\|x-x_{1}\right\|
$$

If follows from (xifi") that

$$
\left\|x_{1}-A x\right\|<\left\|x-x_{1}\right\|=\left\|x_{1}-x_{2}\right\| / 2
$$


As $X$ is strictly convex, $x_{1}-A x$ and hence $A x$, must lie on the line segment joinging $x_{1}$ and $x_{2}$. The above inequalities imply that $A$ is the midpoint, i.e. $A x=x$. Further, $x=T x$ from ( $x v)$ and using ( $x i 1)$, it is easily seen that $x=B x$. Therefore $x$ is in $F$ and hence $F$ is midpoint convex. Since $F$ is closed, it is convex.

REMARK 13. An analogous result can be obtained as noted in Remark 8 . Results similar to Theorem 8 , but established under different contractive conditions, can be found in [1] and [6].

\section{ACKNOWLEDGEMENT}

The first author thanks Profesor G. Jungck for providing him with a preprint of [4].

\section{REFERENCES}

1. RHOADES, B.E. SESSA, S., KHAN, M.S. and KHAN, M.D., "Some fixed point theorems for Hardy-Rogers type mappings", Internat. J. Math. Math. Sc1. 7 (1) (1984), 75-87.

2. HARDY, G.E. and ROGERS, T.D. "A generalization of a fixed point theorem of Reich", Canad. Math. Bul1. 16 (1973), 201-206.

3. JUNGCK, G. "Commuting mappings and fixed points", Amer. Math. Monthly 83 (1976), 261-263.

4. JUNGCK, G. "Compatible mappings and common fixed points", Internat. J. Math. Math. Sc1. 9 (4) (1986), 771-779.

5. SESSA, S. "On a weak commutativity condition in fixed point considerations", Pub1. Inst. Math. 32 (46) (1982), 175-180.

6. FISHER, B. "Common fixed points of four mappings", Bull. Inst. Math. Acad. Sinica 11 (1983), 103-113.

7. DAS, K.M. and NAIK, K.V. "Common fixed point theorems for commuting maps on a metric space", Proc. Amer. Math. Soc. 77 (1979), 369-373.

8. FISHER, B. "Mappings with a common fixed point", Math. Sem. Notes Kobe Univ. 7 (1979), 81-84.

9. FISHER, B. "An addendum to Mappings with a common fixed point", Math. Sem. Notes Kobe Univ. 8 (1980), 513-514.

10. MASSA, S. "Generalized contractions in metric space", Bo11. Un. Mat. Ital. (4) 10 (1974), 689-694.

11. CHANG, S.S. "On common fixed point theorem for a family of $\Phi$-contraction mappings", Math. Japon. 29 (1984), 527-536.

12. FISHER, B. and SESSA, S. "Common fixed points of weakly commuting mappings", Bo11. Polish Acad. Sci., to appear.

13. KASAHARA, S. and SINGH, S.L. "On some recent results on common fixed points", Indian J. Pure App1. Math. 13 (1982), 757-761.

14. NAIDU, S.V.R. and RAJENDRAPRASAD, J. "Common fixed points for four selfmaps on a metric space", Indian J. Pure Appl. Math. 16 (10) (1985), 10891103. 
15. RHOADES, B.E. and SESSA, S. "Common fixed point theorems for three mappings under a weak commutativity condition", Indian J. Pure App1. Math. 17 (1) $(1986), 47-57$.

16. RHOADES, B.E., SESSA, S., KHAN, M.S. and SWALEH, M. "On fixed points of as ymptotically regular mappings", J. Austral. Math. Soc., to appear. 


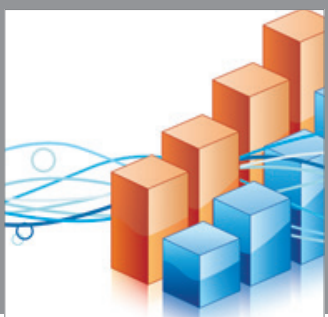

Advances in

Operations Research

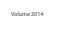

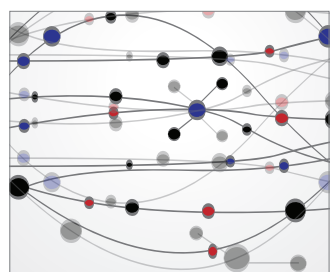

\section{The Scientific} World Journal
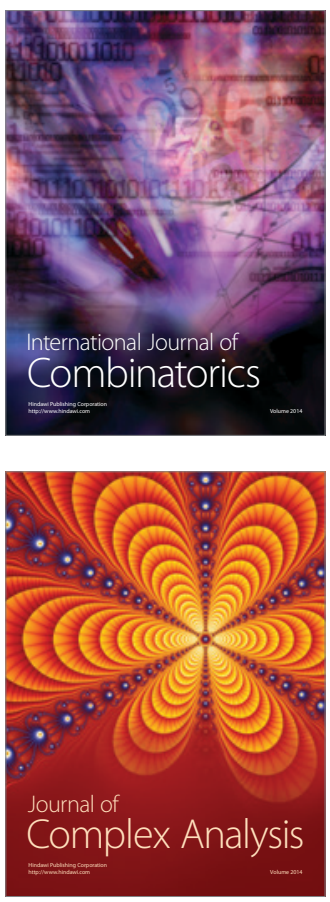

International Journal of

Mathematics and

Mathematical

Sciences
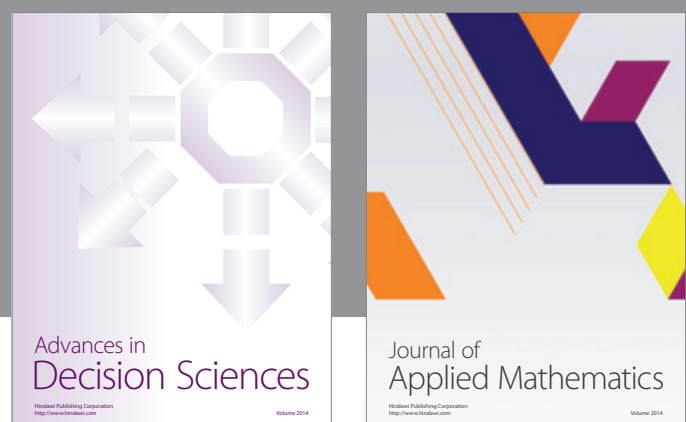

Journal of

Applied Mathematics
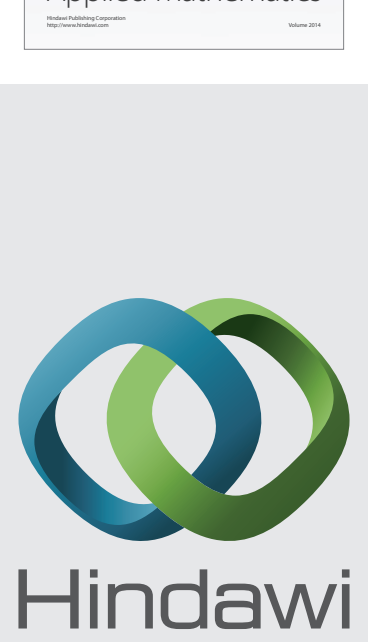

Submit your manuscripts at http://www.hindawi.com
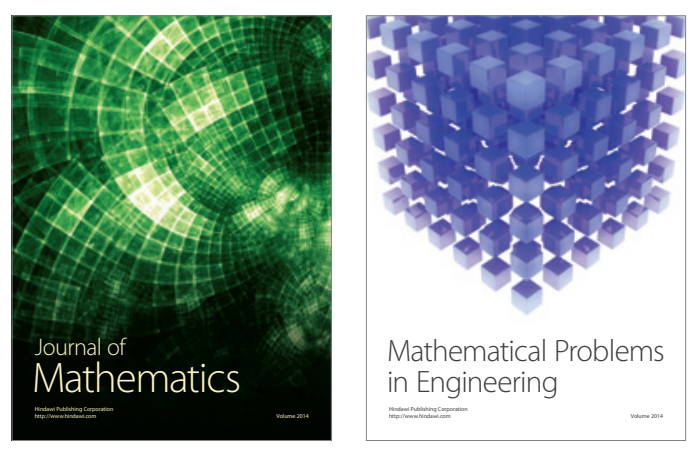

Mathematical Problems in Engineering
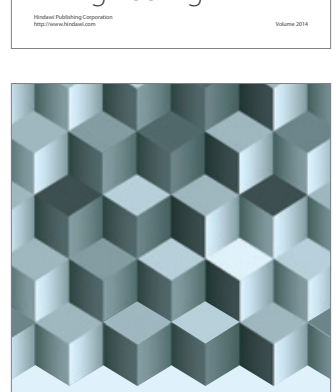

Journal of

Function Spaces
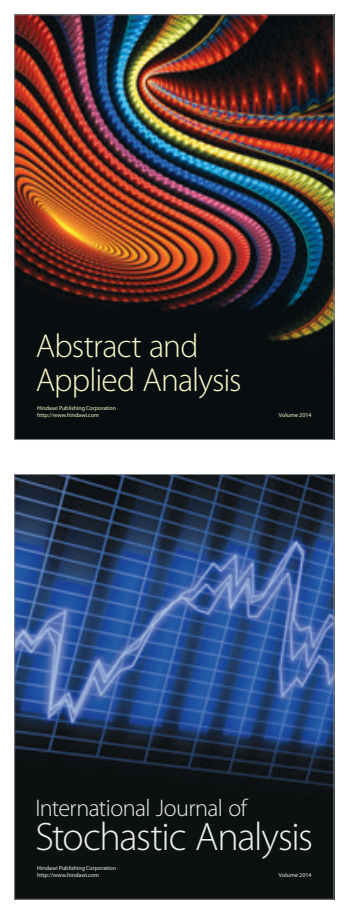

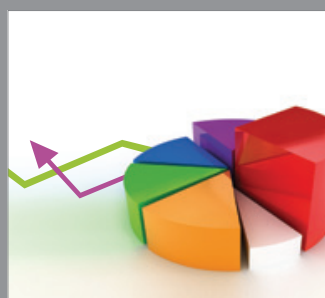

ournal of

Probability and Statistics

Promensencen
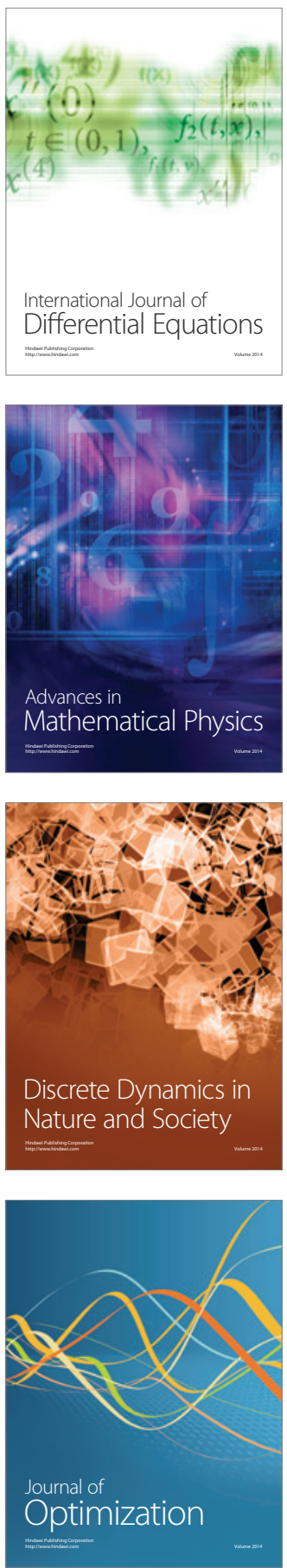